OPEN ACCESS

Edited by:

Caroline Galeotti,

Bicêtre Hospital, France

Reviewed by:

Ozgur Kasapcopur,

Istanbul University-Cerrahpasa, Turkey

Marie-Louise Frémond,

Institut Imagine, France

*Correspondence:

Yasutaka Kuniyoshi

yasutakakuniyoshi@yahoo.co.jp

Specialty section: This article was submitted to

Pediatric Rheumatology,

a section of the journal

Frontiers in Pediatrics

Received: 09 June 2020 Accepted: 09 November 2020 Published: 03 December 2020

Citation:

Kuniyoshi Y, Tokutake H, Takahashi N, Kamura A, Yasuda S and Tashiro $M$

(2020) Comparison of Machine Learning Models for Prediction of Initial Intravenous Immunoglobulin

Resistance in Children With Kawasaki Disease. Front. Pediatr. 8:570834. doi: 10.3389/fped.2020.570834

\section{Comparison of Machine Learning Models for Prediction of Initial Intravenous Immunoglobulin Resistance in Children With Kawasaki Disease}

\author{
Yasutaka Kuniyoshi*, Haruka Tokutake, Natsuki Takahashi, Azusa Kamura, Sumie Yasuda \\ and Makoto Tashiro
}

Department of Pediatrics, Tsugaruhoken Medical COOP Kensei Hospital, Hirosaki, Japan

We constructed an optimal machine learning (ML) method for predicting intravenous immunoglobulin (IVIG) resistance in children with Kawasaki disease (KD) using commonly available clinical and laboratory variables. We retrospectively collected 98 clinical records of hospitalized children with KD (2-109 months of age). We found that 20 (20\%) children were resistant to initial IVIG therapy. We trained three ML techniques, including logistic regression, linear support vector machine, and extreme gradient boosting with 10 variables against IVIG resistance. Moreover, we estimated the predictive performance based on nested 5-fold cross-validation (CV). We also selected variables using the recursive feature elimination method and performed the nested 5-fold CV with selected variables in a similar manner. We compared $\mathrm{ML}$ models with the existing system regardless of their predictive performance. Results of the area under the receiver operator characteristic curve were in the range of 0.58-0.60 in the all-variable model and $0.60-0.75$ in the select model. The specificities were more than 0.90 and higher than those in existing scoring systems, but the sensitivities were lower. Three ML models based on demographics and routine laboratory variables did not provide reliable performance. This is possibly the first study that has attempted to establish a better predictive model. Additional biomarkers are probably needed to generate an effective prediction model.

Keywords: area under the curve, extreme gradient boosting, support vector machine, logistic regression, nested cross-validation, predictive model

\section{INTRODUCTION}

In developed countries, Kawasaki disease (KD) is the major cause of acquired heart disease in children (1). The main complication of KD is coronary artery abnormality (CAA) due to systemic vasculitis (1). The effectiveness of high-dose intravenous immunoglobulin (IVIG) therapy has been established as an initial KD treatment (2). However, approximately $10-20 \%$ children with KD are 
refractory to this treatment and develop persistent or recurrent fever after initial IVIG therapy $(3,4)$. IVIG resistance is a risk factor for the occurrence of CAA (5). Moreover, the development of a more effective treatment options has been challenging. The American Heart Association has reported that patients who were predicted to be at a high risk for development of CAA may benefit from primary adjunctive therapy such as IVIG and corticosteroids (2). Therefore, developing a reliable tool for predicting IVIG resistance is important to reduce the occurrence of CAA.

Several scoring systems (6-12) have been proposed. However, the predictive capacity of the existing scoring systems may not be sufficient, and some scoring systems have poor predictive performance for external datasets (1315). Machine learning (ML) techniques have been applied to clinical diagnosis and prognosis prediction in many fields of medicine (16). To the best of our knowledge, few studies have applied ML methods for predicting resistance to initial IVIG therapy in patients with KD (17). We aimed to construct an optimal ML method for predicting IVIG resistance in children with $\mathrm{KD}$ using commonly available clinical and laboratory variables.

\section{MATERIALS AND METHODS}

\section{Patients and Data Collection}

We retrospectively collected clinical records of patients with KD who were diagnosed based on the Japanese diagnostic guidelines for $\mathrm{KD}$ (18) and hospitalized at Tsugaruhoken Medical COOP Kensei Hospital between January 2010 and October 2019. Patients diagnosed with KD presented with minimum five of the six major symptoms, including fever. Patients with only four or less major symptoms and those with CAA were not included. We excluded children who received initial IVIG treatment $\geq 10$ days after the onset and children administered initial doses of $<2 \mathrm{~g} / \mathrm{kg} /$ day. We defined the first illness day as the first day on which a patient had fever. We defined a responder as a patient whose temperature had decreased to $<37.5^{\circ} \mathrm{C}$ within $36 \mathrm{~h}$ after initial IVIG treatment $(9,15)$.

We collected the following data before the initial IVIG treatment: months of age, gender, illness days with IVIG administration, white blood cell count (WBC), neutrophil percentage, hematocrit (Ht), platelet count (PLT), aspartate aminotransferase (AST), alanine aminotransferase (ALT), total bilirubin (TBil), and sodium $(\mathrm{Na})$, albumin (Alb), and C-reactive protein (CRP) levels. All these variables were available before treatment.

We defined coronary arteries as abnormal when the luminal diameters were more than $3.0 \mathrm{~mm}$ in children younger than

\footnotetext{
Abbreviations: Alb, albumin; ALT, alanine aminotransferase; AST, aspartate aminotransferase; AUC, area under the receiver operator characteristic curve; CAA, coronary artery abnormality; CRP, C-reactive protein; CV, cross-validation; Ht, hematocrit; IVIG, intravenous immunoglobulin; KD, Kawasaki disease; LR, logistic regression; ML, machine learning; Na, sodium; PLT, platelet count; SVM, support vector machine; TBil, total bilirubin; WBC, white blood cell count; XGB, eXtreme gradient boosting.
}

5 years or more than $4.0 \mathrm{~mm}$ in those 5 years and older, when the internal diameter of a segment was 1.5 times or greater than that of an adjacent segment, or when the luminal contour was evidently irregular (19). We recorded the maximum coronary artery diameter within 1 month after the onset of the disease.

\section{Statistical Analysis}

We performed statistical analyses using Python version 3.6 (Python Software Foundation). We applied Mann-Whitney $U$-tests for continuous variables and Chi-square tests for categorical variables.

We evaluated the predictive performance of the three supervised ML classifiers and existing scoring systems. We trained logistic regression (LR) with L2 regularization, linear support vector machine (SVM), and eXtreme gradient boosting (XGB) models to predict IVIG resistance, using scikitlearn and XGBoost packages. We evaluated the predictive performance based on sensitivity, specificity, and area under the receiver operator characteristic curve (AUC). We produced three ML models with 10 variables that did not contain missing values (months of age, gender, illness days with IVIG administration, WBC, Ht, PLT, AST, Na, Alb, and CRP).

To evaluate the predictive performance of the three ML models and algorithms, we used the nested 5-fold crossvalidation (CV) approach (20) with GridSearchCV for hyperparameter optimization. We applied a nested $\mathrm{CV}$ procedure to estimate an unbiased generalization performance of $\mathrm{ML}$ algorithms (21). The two CV cycles included an inner loop for tuning hyper-parameters and outer loop for estimating performance in nested CV (Figure 1). First, the original dataset was divided into five data folds with approximately equal numbers of respondent and non-respondent cases. One data fold was reserved for test fold. The remaining four data folds (training folds) were passed to the inner loop. The inner loop performed 5 -fold CVs to identify the best hyper-parameter combination. We selected the hyper-parameter combination that maximized each performance metrics over all steps of the inner loop. In LR and linear SVM models, the penalty parameter $C$ was explored in $[0.01,0.1,1,10$, and 100]. In XGB model, the maximum depth of a tree (max_depth), the minimum sum of instance weight needed in a child (min_child_weight), and gamma were explored in $[3,5],[1,2,3]$, and $[0,3,10]$, respectively. For the other hyper-parameters, we used default values of the scikit-learn method. These were tuned by testing all possible hyper-parameter combinations in the inner $\mathrm{CV}$. We then trained our model on training folds using the best hyper-parameter combination; thereafter, we evaluated model performance on the test fold. This process was repeated five times, once for each iteration of the outer loop. Finally, we calculated the average performance over 5-folds. We also repeated nested CVs 10 times in separate splits and derived the average of the results to avoid sampling bias and data overfitting.

Additionally, we selected variables using the recursive feature elimination method. Then, we performed a nested 5-fold CV with selected variables in a similar manner. In all, we have constructed 


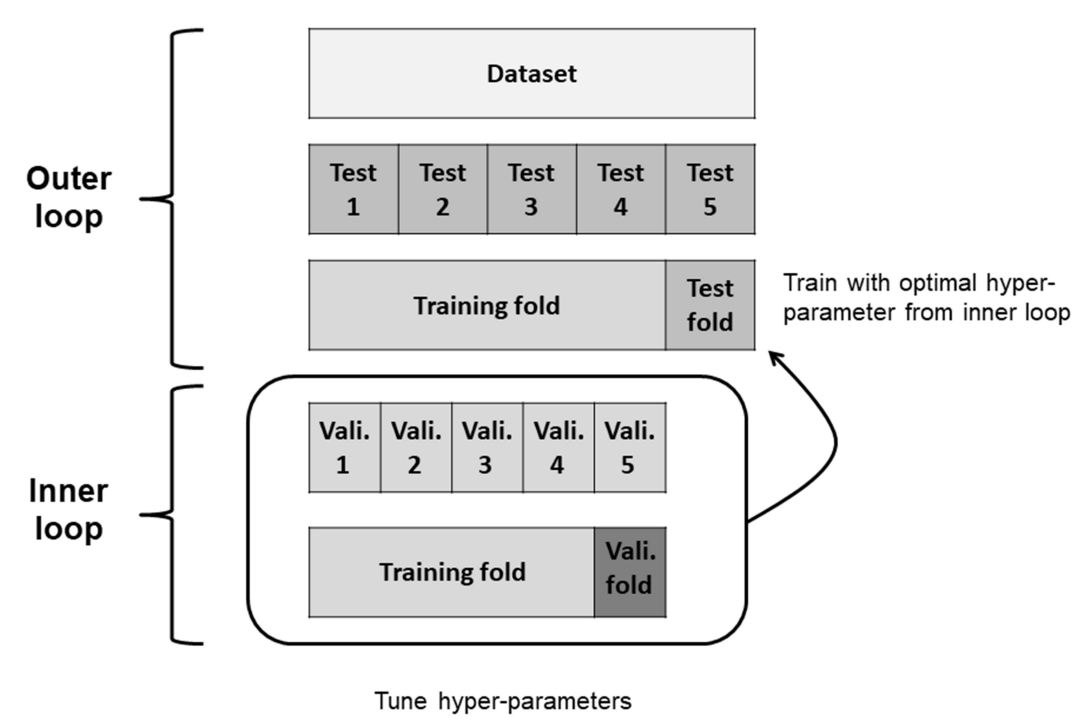

FIGURE 1 | Flow chart of the 5 -fold nested cross-validation. Vali., validation.

and then evaluated two types of models: all-variable model and select-variable model.

\section{RESULTS}

\section{Characteristics of Patients}

We collected data from 109 children with KD treated at our hospital. We excluded data from 11 children because 9 children had received initial IVIG at $<2 \mathrm{~g} / \mathrm{kg} /$ day and 4 had received initial IVIG treatment $\geq 10$ days after the onset of the disease. Consequently, we statistically analyzed data from 98 children aged 2-109 months. Table 1 summarizes the demographic and laboratory data of patients. Among them, 20 (20\%) children were resistant to the initial IVIG therapy. Only the AST and ALT levels were significantly higher in the IVIG-responsive group than in the IVIG resistant group. The proportion of CAA in the IVIG resistant group was higher than that in the IVIGresponsive group.

\section{Predictive Performance of the ML Model}

As shown in Table 2, the AUCs of the all-variable models were $0.58-0.60$ in all models, and those of the select-variable models were $0.60-0.75$. The results on specificity and accuracy were $0.94-$ 0.99 and $0.78-0.79$ in the all-variable models, and $0.96-1.00$ and $0.78-0.80$ in the select-variable models. The results of specificity and accuracy were high, but those on sensitivity were all lower.

\section{DISCUSSION}

We retrospectively evaluated the performances of three $\mathrm{ML}$ models to predict the resistance to initial IVIG therapy in a single-center pediatric population of KD. Our results revealed that the three ML models based on demographics and routine laboratory variables did not perform reliably.
TABLE 1 | Comparison of clinical and laboratory characteristics in IVIG-responsive and -resistant patients.

\begin{tabular}{|c|c|c|c|}
\hline & $\begin{array}{l}\text { Responsive } \\
\quad(n=78)\end{array}$ & $\begin{array}{l}\text { Resistant } \\
(n=20)\end{array}$ & $P$-value \\
\hline Age, months of age, median (IQR) & $22(9-37)$ & $26(17-30)$ & 0.49 \\
\hline $\begin{array}{l}\text { Illness days with IVIG administration, } \\
\text { days, median (IQR) }\end{array}$ & $5(4-6)$ & $4(3.8-5)$ & 0.16 \\
\hline Gender, male, $n(\%)$ & $40(51)$ & $13(65)$ & 0.40 \\
\hline $\begin{array}{l}\text { White blood cell count, } \times 10^{2} / \mathrm{mm}^{3} \text {, } \\
\text { median (IQR) }\end{array}$ & $\begin{array}{c}151 \\
(121-175)\end{array}$ & $\begin{array}{c}144 \\
(113-179)\end{array}$ & 0.96 \\
\hline Neutrophil, \%, median (IQR) & $66(59-76)$ & $73(67-79)$ & 0.12 \\
\hline Hematocrit, \%, median (IQR) & $34(32-36)$ & $35(33-36)$ & 0.65 \\
\hline $\begin{array}{l}\text { Platelet count, } \times 10^{4} / \mathrm{mm}^{3} \text {, median } \\
\text { (IQR) }\end{array}$ & $35(28-42)$ & $32(27-38)$ & 0.41 \\
\hline $\begin{array}{l}\text { Aspartate aminotransferase, IU/L, } \\
\text { median (IQR) }\end{array}$ & $30(24-43)$ & $96(34-308)$ & $<0.001$ \\
\hline $\begin{array}{l}\text { Alanine aminotransferase, IU/L, } \\
\text { median (IQR) }\end{array}$ & $20(12-32)$ & 75 (20-232) & 0.004 \\
\hline Total bilirubin, mg/dl, median (IQR) & $\begin{array}{c}0.53 \\
(0.41-0.69)\end{array}$ & $\begin{array}{c}0.81 \\
(0.50-1.37)\end{array}$ & 0.36 \\
\hline Sodium, mmol/L, median (IQR) & $\begin{array}{c}133 \\
(131-134)\end{array}$ & $\begin{array}{c}132 \\
(131-134)\end{array}$ & 0.88 \\
\hline Albumin, g/dl, median (IQR) & $3.3(3.1-3.6)$ & $3.4(3.1-3.6)$ & 0.85 \\
\hline $\begin{array}{l}\text { C-reactive protein, mg/dl, median } \\
\text { (IQR) }\end{array}$ & $6.3(3.8-9.3)$ & $7.4(5.3-10.6)$ & 0.26 \\
\hline Coronary artery abnormalities, $n$ (\%) & $5(6.4)$ & $6(30)$ & $\times 0.007$ \\
\hline
\end{tabular}

IVIG, intravenous immunoglobulin; SD, standard deviation; IQR; interquartile range. Data are analyzed by Mann-Whithey U tests for continuous variables and Chi-square tests for categorical variables.

Different clinical scoring systems have been established to predict IVIG resistance, including those by Kobayashi et al. (6), Egami et al. (7), and Sano et al. (8) in Japan. The sensitivities 
TABLE 2 | Prediction performances of the three machine learning models and existing scoring systems.

\begin{tabular}{|c|c|c|c|c|c|c|c|}
\hline & & & Feature & AUC & Sensitivity & Specificity & Accuracy \\
\hline \multirow[t]{3}{*}{ All-variable model } & LR & & All 10 variables & $0.59 \pm 0.052$ & $0.22 \pm 0.055$ & $0.94 \pm 0.017$ & $0.79 \pm 0.021$ \\
\hline & Linear SVM & & All 10 variables & $0.58 \pm 0.040$ & $0.20 \pm 0.059$ & $0.95 \pm 0.014$ & $0.79 \pm 0.018$ \\
\hline & XGBoost & & All 10 variables & $0.60 \pm 0.048$ & $0.26 \pm 0.095$ & $0.99 \pm 0.021$ & $0.78 \pm 0.026$ \\
\hline \multirow[t]{9}{*}{ Select-variable model } & LR & Model 1 & AST & $0.75 \pm 0.011$ & $0.15 \pm 0.039$ & $0.97 \pm 0.006$ & $0.79 \pm 0.012$ \\
\hline & & Model 2 & WBC, AST & $0.67 \pm 0.027$ & $0.16 \pm 0.037$ & $0.97 \pm 0.008$ & $0.80 \pm 0.011$ \\
\hline & & Model 3 & Day, WBC, PLT, AST, CRP & $0.67 \pm 0.022$ & $0.19 \pm 0.049$ & $0.96 \pm 0.049$ & $0.80 \pm 0.010$ \\
\hline & SVM & Model 1 & AST & $0.75 \pm 0.011$ & $0.14 \pm 0.037$ & $0.96 \pm 0.012$ & $0.79 \pm 0.015$ \\
\hline & & Model 2 & WBC, Ht, PLT, AST & $0.66 \pm 0.035$ & $0.16 \pm 0.039$ & $0.97 \pm 0.010$ & $0.80 \pm 0.010$ \\
\hline & & Model 3 & WBC, AST & $0.68 \pm 0.024$ & $0.14 \pm 0.035$ & $0.97 \pm 0.006$ & $0.79 \pm 0.007$ \\
\hline & XGBoost & Model 1 & $\mathrm{Na}, \mathrm{AST}$ & $0.65 \pm 0.032$ & $0.28 \pm 0.078$ & $1.00 \pm 0.008$ & $0.78 \pm 0.021$ \\
\hline & & Model 2 & Age, Day, Ht, Na, AST, CRP & $0.61 \pm 0.036$ & $0.31 \pm 0.073$ & $0.99 \pm 0.008$ & $0.79 \pm 0.025$ \\
\hline & & Model 3 & Age, Day, Ht, Na, AST, Alb, CRP & $0.60 \pm 0.035$ & $0.33 \pm 0.078$ & $0.99 \pm 0.008$ & $0.79 \pm 0.022$ \\
\hline \multirow[t]{3}{*}{ Existing scoring systems } & \multicolumn{3}{|c|}{ Kobayashi (8) system $(n=93)$} & NA & 0.70 & 0.62 & 0.63 \\
\hline & \multicolumn{3}{|c|}{ Egami (9) system ( $n=98)$} & NA & 0.55 & 0.81 & 0.76 \\
\hline & \multicolumn{3}{|c|}{ Sano (10) system $(n=62)$} & NA & 0.41 & 0.96 & 0.81 \\
\hline
\end{tabular}

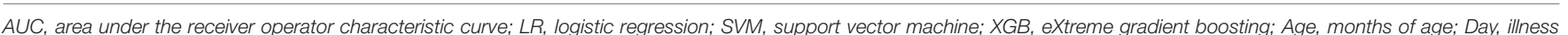

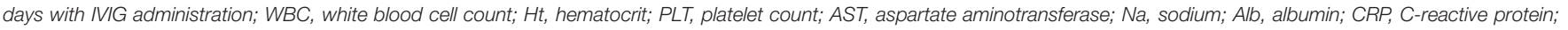
NA, not applicable.

and specificities of those systems were reported to be $0.76-0.78$ and $0.76-0.86$ in the original studies. However, almost all clinical scores published are limited in their predictive capacity. Similar predictive accuracies were not achieved in other populations (13-15). As shown in Table 2, the existing scoring systems also did not achieve a good prediction against our dataset.

Additional clinical information may be needed to improve the prediction model. Owing to the similarity of each clinical and laboratory characteristics between IVIG-responsive and resistant patients in the current dataset, neither our model nor the existing model may have performed reliably. There may be a need to construct and evaluate new models that also incorporate clinical major symptoms (10) and/or other laboratory data such as erythrocyte sedimentation rate (10) or N-terminal pro-brain natriuretic peptide (22).

Our prediction models using three ML techniques have equally less reliable performance as the existing scoring systems; particularly, the sensitivity were low in all ML algorithms. Our results serve as a first step to establish a good prediction tool. Feature engineering or ensemble learning, which combines several ML techniques into one predictive model, may help improve performance. Alternatively, ML models have advantages over the existing prediction scoring systems. The predictive performances of scoring systems could differ depending on countries or ethnicities $(11,13,23)$. ML is flexible and can be suitable for many tasks. Therefore, the ML approach makes it easy for the model to retrain and update the using the newest data.

To our best knowledge, this is the first study to compare the performances of ML methods for predicting IVIG resistance. There is a study which was designed to develop the prediction model using random forest (17). However, validation procedures were not conducted, though the performance was excellent.
Conversely, there are certain limitations. First, the dataset was relatively small. However, we used nested CVs to obtain unbiased estimates of the true error. We also repeated the nested CVs 10 times and averaged the validation error to reduce sampling bias. Nested CV can choose the classification model by obtaining reliable classification performance and avoiding overfitting (24). Second, the present study was conducted based on dataset derived from a single center. Accordingly, our results may not apply to other populations. However, we consider it meaningful to rebuild the model, similarly using the center's original data. Third, this is a retrospective study. We need to perform a combined analysis of three ML models on a prospective basis.

In conclusion, we evaluated the performance of ML models for predicting resistance to IVIG therapy in children with KD. However, our three ML models based only on demographics and routine laboratory variables did not provide reliable performances. Further studies are needed to improve predictive models. Additional biomarkers are likely to be needed to generate an effective prediction model.

\section{DATA AVAILABILITY STATEMENT}

The raw data supporting the conclusions of this article will be made available by the authors, Yasutaka Kuniyoshi, upon reasonable request.

\section{ETHICS STATEMENT}

The studies involving human participants were reviewed and approved by Ethics Committee of Tsugaruhoken Medial COOP Kensei Hospital. Written informed consent from the participants' legal guardian/next of kin was not required to participate in 
this study in accordance with the national legislation and the institutional requirements.

\section{AUTHOR CONTRIBUTIONS}

YK designed the study, drafted the manuscript, performed the statistical analysis, and interpreted the results. All authors have

\section{REFERENCES}

1. Gordon JB, Kahn AM, Burns JC. When children with Kawasaki disease grow up: myocardial and vascular complications in adulthood. J Am Coll Cardiol. (2009) 54:1911-20. doi: 10.1016/j.jacc.2009.04.102

2. McCrindle BW, Rowley AH, Newburger JW, Burns JC, Bolger AF, Gewitz $M$, et al. Diagnosis, treatment, and long-term management of Kawasaki disease: a scientific statement for health professionals from the American Heart Association. Circulation. (2017) 135:e92799. doi: 10.1161/CIR.0000000000000484

3. Bar-Meir M, Kalisky I, Schwartz A, Somekh E, Tasher D, Israeli Kawasaki Group. Prediction of resistance to intravenous immunoglobulin in children with Kawasaki disease. J Pediatr Infect Dis Soc. (2018) 7:259. doi: 10.1093/jpids/piw075

4. Kido S, Ae R, Kosami K, Matsubara Y, Makino N, Sasahara T, et al. Seasonality of i.v. immunoglobulin responsiveness in Kawasaki disease. Pediatr Int. (2019) 61:539-43. doi: 10.1111/ped.13863

5. Kibata T, Suzuki Y, Hasegawa S, Matsushige T, Kusuda T, Hoshide M, et al. Coronary artery lesions and the increasing incidence of Kawasaki disease resistant to initial immunoglobulin. Int J Cardiol. (2016) 214:20915. doi: 10.1016/j.ijcard.2016.03.017

6. Kobayashi T, Inoue $\mathrm{Y}$, Takeuchi K, Okada Y, Tamura K, Tomomasa $\mathrm{T}$, et al. Prediction of intravenous immunoglobulin unresponsiveness in patients with Kawasaki disease. Circulation. (2006) 113:2606-12. doi: 10.1161/CIRCULATIONAHA.105.592865

7. Egami $K$, Muta $H$, Ishii $M$, Suda $K$, Sugahara $Y$, Iemura $M$, et al. Prediction of resistance to intravenous immunoglobulin treatment in patients with Kawasaki disease. J Pediatr. (2006) 149:237-40. doi: 10.1016/j.jpeds.2006.03.050

8. Sano T, Kurotobi S, Matsuzaki K, Yamamoto T, Maki I, Miki K, et al. Prediction of non-responsiveness to standard high-dose gamma-globulin therapy in patients with acute Kawasaki disease before starting initial treatment. Eur J Pediatr. (2007) 166:131-7. doi: 10.1007/s00431-006-0223-z

9. Tang Y, Yan W, Sun L, Huang J, Qian W, Ding Y, et al. Prediction of intravenous immunoglobulin resistance in Kawasaki disease in an East China population. Clin Rheumatol. (2016) 35:2771-6. doi: 10.1007/s10067-016-3370-2

10. Li X, Chen $\mathrm{Y}$, Tang $\mathrm{Y}$, Ding $\mathrm{Y}, \mathrm{Xu} \mathrm{Q}$, Sun $\mathrm{L}$, et al. Predictors of intravenous immunoglobulin-resistant Kawasaki disease in children: a meta-analysis of 4442 cases. Eur J Pediatr. (2018) 177:1279-92. doi: 10.1007/s00431-018-3182-2

11. Yang S, Song R, Zhang J, Li X, Li C. Predictive tool for intravenous immunoglobulin resistance of Kawasaki disease in Beijing. Arch Dis Child. (2019) 104:262-7. doi: 10.1136/archdischild-2017-314512

12. Tan XH, Zhang XW, Wang XY, He XQ, Fan C, Lyu TW, et al. A new model for predicting intravenous immunoglobin-resistant Kawasaki disease in Chongqing: a retrospective study on 5277 patients. Sci Rep. (2019) 9:1722. doi: 10.1038/s41598-019-39330-y

13. Sleeper LA, Minich LL, McCrindle BM, Li JS, Mason W, Colan $\mathrm{SD}$, et al. Evaluation of Kawasaki disease risk-scoring systems for intravenous immunoglobulin resistance. J Pediatr. (2011) 158:831-5.e3. doi: 10.1016/j.jpeds.2010.10.031 read and approved the final manuscript and contributed to data collection.

\section{ACKNOWLEDGMENTS}

The authors would like to thank Enago (www.enago.jp) for the English language review.

14. Song R, Yao W, Li X. Efficacy of four scoring systems in predicting intravenous immunoglobulin resistance in children with Kawasaki disease in a children's hospital in Beijing, North China. J Pediatr. (2017) 184:1204. doi: 10.1016/j.jpeds.2016.12.018

15. Qian W, Tang Y, Yan W, Sun L, Lv H. A comparison of efficacy of six prediction models for intravenous immunoglobulin resistance in Kawasaki disease. Ital J Pediatr. (2018) 44:33. doi: 10.1186/s13052-018-0475-z

16. Rajkomar A, Dean J, Kohane I. Machine learning in medicine. N Engl J Med. (2019) 380:1347-58. doi: 10.1056/NEJMra1814259

17. Takeuchi M, Inuzuka R, Hayashi T, Shindo T, Hirata Y, Shimizu N, et al. Novel risk assessment tool for immunoglobulin resistance in Kawasaki disease: application using a random forest classifier. Pediatr Infect Dis J. (2017) 36:821-6. doi: 10.1097/INF.0000000000001621

18. Ayusawa M, Sonobe T, Uemura S, Ogawa S, Nakamura Y, Kiyosawa N, et al. Revision of diagnostic guidelines for Kawasaki disease (the 5th revised edition). Pediatr Int. (2005) 47:232-4. doi: 10.1111/j.1442-200x.2005.02033.x

19. Kobayashi T, Saji T, Otani T, Takeuchi K, Nakamura T, Arakawa H, et al. Efficacy of immunoglobulin plus prednisolone for prevention of coronary artery abnormalities in severe Kawasaki disease (RAISE study): a randomised, open-label, blinded-endpoints trial. Lancet. (2012) 379:161320. doi: 10.1016/S0140-6736(11)61930-2

20. Masino AJ, Harris MC, Forsyth D, Ostapenko S, Srinivasan L, Bonafide CP, et al. Machine learning models for early sepsis recognition in the neonatal intensive care unit using readily available electronic health record data. PLoS ONE. (2019) 14:e0212665. doi: 10.1371/journal.pone.0212665

21. Varma S, Simon R. Bias in error estimation when using crossvalidation for model selection. BMC Bioinformatics. (2006) 7:91. doi: 10.1186/1471-2105-7-91

22. Shao S, Luo $\mathrm{C}$, Zhou $\mathrm{K}$, Hua $\mathrm{Y}$, Wu M, Liu L, et al. The role of agespecific $\mathrm{N}$-terminal pro-brain natriuretic peptide cutoff values in predicting intravenous immunoglobulin resistance in Kawasaki disease: a prospective cohort study. Pediatr Rheumatol. (2019) 17:65. doi: 10.1186/s12969-0190368-8

23. Sánchez-Manubens J, Antón J, Bou R, Iglesias E, Calzada-Hernandez J, Borlan $\mathrm{S}$, et al. Role of the Egami score to predict immunoglobulin resistance in Kawasaki disease among a western Mediterranean population. Rheumatol Int. (2016) 36:905-10. doi: 10.1007/s00296-016-3499-y

24. Cawley GC, Talbot NLC. On over-fitting in model selection and subsequent selection bias in performance evaluation. J Mach Learn Res. (2010) 11:2079107. doi: $10.5555 / 1756006.1859921$

Conflict of Interest: The authors declare that the research was conducted in the absence of any commercial or financial relationships that could be construed as a potential conflict of interest.

Copyright $\odot 2020$ Kuniyoshi, Tokutake, Takahashi, Kamura, Yasuda and Tashiro. This is an open-access article distributed under the terms of the Creative Commons Attribution License (CC BY). The use, distribution or reproduction in other forums is permitted, provided the original author(s) and the copyright owner(s) are credited and that the original publication in this journal is cited, in accordance with accepted academic practice. No use, distribution or reproduction is permitted which does not comply with these terms. 